\title{
Enhancing Guaranteed Delays with Network Coding
}

\author{
Ali Mahmino ${ }^{1}$, Jérôme Lacan ${ }^{2}$, and Christian Fraboul ${ }^{3}$ \\ ${ }^{1}$ ENSICA/IRIT/INPT/ENSEEIHT, University of Toulouse \\ 1, place E. Blouin, 31056 Toulouse Cedex, France \\ Ali.Mahmino@ensica.fr \\ ${ }^{2}$ LAAS-CNRS/ENSICA, University of Toulouse \\ jerome.lacan@ensica.fr \\ ${ }^{3}$ IRIT/INPT/ENSEEIHT, University of Toulouse \\ Christian.Fraboul@enseeiht.fr
}

\begin{abstract}
For networks providing QoS guarantees, this paper determines and evaluates the worst case end-to-end delays for strategies based on network coding and multiplexing. It is shown that the end-to-end delay does not depend on the same parameters with the two strategies. This result can be explained by the fact that network coding can cope with congestions better than classical routing because it processes simultaneously packet from different flows. In counterpart, additional delays like algebraic combinations of packets are added.
\end{abstract}

Keywords: Network Coding, network calculus, worst-case delays, buffering.

\section{Introduction}

In networks providing quality of service (QoS) guarantees (ATM, IntServ, DiffServ, .. . ) input data flows verify constraints of burstiness and maximal throughput. On the other hand, the network ensures a level of quality of service (QoS) guarantee expressed in terms of end-to-end delays or minimal throughput. The different guarantees and constraints characterizing the network and the flows can be represented by using network calculus. Network Calculus is a framework providing deterministic bounds of QoS parameters such as end-to-end delays or backlogs by using Min-Plus algebra. This theory was introduced and developed in [1] by generalizing previous works such as 2].

In this paper, we focus on decreasing worst case end-to-end delays by using network coding techniques. Network coding is a concept introduced by Ahlswede et al. in 3], consisting in combining packets in routing nodes instead of simply forwarding them as a classical routing strategy. In addition to the optimization of the utilization of the capacity of the network, this technique also allows to decrease average end-to-end delays. For a complete file, the evaluation of the delay performance gains of several network coding strategies was studied in 5] in the context of a downlink transmission of files from a single base station to 
multiple receivers. In [4], a queuing model introduced for network coding allows to estimate the average end-to-end delays of the packets for independent Poisson flows in whole networks.

The problematic considered here concerns also end-to-end delays like in 4], but the flows are constrained and the network provide QoS guarantees. In this context, our main objective is to reduce the worst cases delays.

The main idea is based on the fact that, with network coding, the node processes several packets simultaneously. Compared to a classical routing approach dealing with packets in sequence, this allows to reduce the maximum time spent by packets in the buffers. In counterpart, other delays are added like the time needed to achieve the linear combination or the time spent by a packet while it waits the corresponding packets arriving from other links.

The network hypotheses and the network coding node strategy are detailed in Section 2. The QoS guarantees provided by the nodes/network are evaluated and compared with a classical routing strategy in Sections 3 and 4. Finally, Section 5 presents the main conclusions.

\section{Network Hypotheses}

Consider a communication network represented by an acyclic directed graph $G=(V, E)$ with a vertex set $V$ and an edge set $E$. The set of nodes is divided into three categories: source nodes, intermediate nodes and receiver nodes. Each Source node $S_{i}$ generates a flow $F_{i}$ of packets of fixed length $L$ constrained by affine arrival curves $\gamma_{\rho_{i}, \sigma_{i}}$ where $\rho_{i}$ is the leak rate and $\sigma_{i}$ is the burstiness size (see [1]). Intermediate nodes are the nodes situated between the sources and the receiver nodes. The nodes, e.g. $v_{i}$ and $v_{j}$, are connected by directed edges, denoted by $e_{i, j}$, each of them having a given maximum capacity $C_{i, j}$ and a maximum transmission delay $T_{i, j}$. The service provided by the link is represented by a rate latency service curve $\beta_{C_{i, j}, T_{i, j}}(t)$ (see [1]). We assume that all the nodes are synchronized and that each node knows the maximum transmission delays between itself and the neighbors generating its input flows. We suppose that the capacity of every output edge of a node is greater or equal than the sum of capacities of all input edges. We assume that the time necessary to perform a linear combination of several packets does not depend on the number of packets and is upper-bounded by $T_{l c}$. Each intermediate node $N_{n+1}$ offers a service curve $\beta_{C_{n+1}, T_{B_{i}}^{n+1}+T_{l c}+\tau_{n+1}}(t)$ where $T_{B_{i}}^{n+1}$ is the maximum time spent by a packet of flow $i$ in the buffers while waiting for corresponding packets of others flows. $\tau_{n+1}$ is the service delay to transmit a packet. Each multiplexer node $N_{n+1}$ offers a service curve $\beta_{C_{n+1}, \tau_{n+1}}(t)$ where $\tau_{n+1}$ is the service delay to transmit a packet of the aggregate flow and $C_{n+1}$ is the output edge capacity (see Figure 1(a).

The network coding strategy described here is based on a fixed network code determined a priori. To ensure that receivers decode all the received packets, the concept of packet generation is used, where each generation corresponds to a fixed-length time interval $\left[t_{i}, t_{i}+\Delta[\right.$ in which a source generates at most one packet of a flow. The linear network code only combines packets belonging to the 


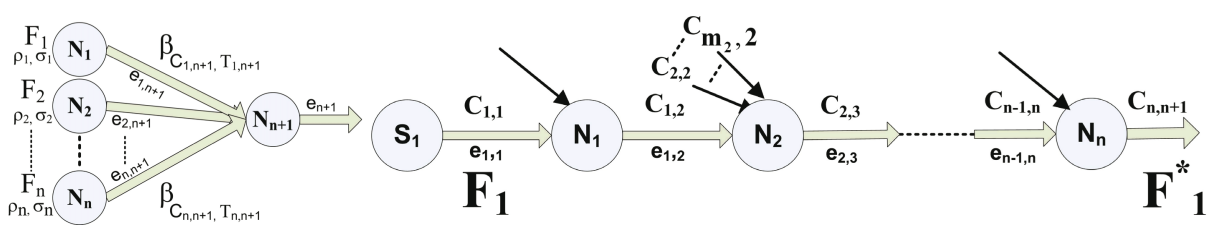

(a) N-flows multiplexer

(b) Transmission of a flow through $N$ nodes

Fig. 1. Node Level and Network Level examples

same generation. To perform a linear combination of several packets, the node must wait all the packets of the same generation. Since the network provides delays guarantees, a node is able to compute a time limit for reception of a given generation. If a packet of a given generation is not received before the time limit, the node assumes that the source did not generate a packet in this time interval, and it performs combinations with the available packets. To evaluate the interest of using network coding compared with a classical routing/multiplexing approach, we proceed in two steps: Node Level and Network Level.

\section{Multiplexing vs. Coding: Delay Analysis at Node Level}

\subsection{Multiplexing Delay}

We suppose that the FIFO multiplexer node $N_{n+1}$ presented on Figure 1(a) offers a service curve $\beta_{C_{n+1}, \tau_{n+1}}$ to the aggregated flow. From [1] and the hypotheses (and notations) introduced in Section 2, it can be shown that the maximum delay experienced by a packet of the flow $F_{i}$ in this multiplexer node is $T_{i}^{m n}=$ $\tau_{n+1}+\left(\sum_{k=1, k \neq i}^{n} \sigma_{k}+\rho_{k} T_{k, n+1}\right) /\left(C_{n+1}\right)+\left(\sigma_{i}+\rho_{i} T_{i, n+1}\right) /\left(C_{n+1}-\sum_{k=1, k \neq i}^{n} \rho_{k}\right)$. Then the arrival curve (see [1]) of the flow $F_{i}$ at the output of the multiplexer is equal to $\gamma_{\rho_{i}, \sigma_{i}^{*}}$ where $\sigma_{i}^{*}=\sigma_{i}+\rho_{i}\left(T_{i, n+1}+\tau_{n+1}+\left(\sum_{k=1, k \neq i}^{n} \sigma_{k}+\rho_{k} T_{k, n+1}\right) /\left(C_{n+1}\right)\right)$.

\subsection{Coding Delay}

Let us now consider the node presented on Figure 1(a) as an intermediate node combining the flows $F_{i}, i=1, \ldots, n$, respectively constrained by $\gamma_{\rho_{i}, \sigma_{i}}$. Following the hypotheses and notations introduced in Section 2 , it can be shown that the maximum delay experienced by a packet of $F_{i}$ in the node is $T_{i}^{n c}=T_{B_{i}}^{n+1}+$ $T_{l c}+\tau_{n+1}+\sigma_{i}+\rho_{i} T_{i, n+1} / C_{n+1}$. Note that this delay corresponds to the delay between an input packet and the corresponding encoded packet. An arrival curve of a subflow corresponding to $F_{i}$ at the output of this intermediate node is $\gamma_{\rho_{i}, \sigma_{i}^{*}}$ where $\sigma_{i}^{*}=\sigma_{i}+\rho_{i} T_{i, n+1}+\rho_{i}\left(T_{B_{i}}^{n+1}+T_{l c}+\tau_{n+1}\right)$. 


\section{Multiplexing vs. Coding: End-to-End Delays Analysis}

The properties of the network calculus facilitate the generalization of the results at node-level to the network level.

From [1] and Section 3.1, it can be shown that a FIFO multiplexer node $N_{k}$ (see Figure 1(b) node $N_{2}$ ) offers to the $i^{\text {th }}$ input flow a service curve $\beta_{C_{k, r}-X_{i}^{k}, \tau_{k}+Y_{i}^{k}}^{i}(t)$ where $X_{i}^{k}=\sum_{s=1, s \neq i}^{m_{k}} C_{s, k}$ and $Y_{i}^{k}=\sum_{s=1, s \neq i}^{m_{k}} \sigma_{s} / C_{k, r}$ and $m_{k}$ is the number of input flows to node $k$ and $C_{k, r}$ is the output edge capacity. The maximum delay of the flow $F_{i}$ from the source to the output of this network is $T_{i}^{m n}=T_{\text {tot }}^{i}+\frac{\sigma_{i}}{C_{\text {tot }}^{i}}$ where $T_{\text {tot }}^{i}=T_{1,1}+\sum_{k=1}^{n-1} T_{k, k+1}+\sum_{k=1}^{n} \tau_{k}+Y_{i}^{k}$ and $C_{\text {tot }}^{i}=\min \left(C_{1,1}, C_{1,2}-X_{1}, C_{2,3}-X_{2}, \cdots, C_{n, n+1}-X_{n}\right)$.

For the coding case, from the results of Section 3.2 the maximum delay of the flow $F_{i}$ from the source to the output of this network is equal to $T_{i}^{c n}=T_{t o t}^{i}+\frac{\sigma_{i}}{C_{t o t}^{2}}$ where $C_{\text {tot }}^{i}=\min \left(C_{1,1}, C_{1,2}, \cdots, C_{n-1, n}\right)$ and $T_{\text {tot }}^{i}=T_{1,1}+\sum_{k=1}^{n-1} T_{k, k+1}+\sum_{k=1}^{n} T_{B_{i}^{k}}+$ $T_{l c}+\tau_{k}$.

Even if the worst case delays of multiplexing and coding strategy are difficult to compare in the general case, the main interest of these formulas is to emphasize the parameters that must be taken into account to decrease the worst case endto-end delays.

\section{Conclusion}

This paper has presented a new interest of network coding. The worst case endto-end delays were determined for classical routing/multiplexing and network coding approaches in networks providing QoS guarantees. These results show that the two formulas do not depend on the same parameters. These results can be viewed as the first step toward the construction of networks using network coding providing optimal level of guaranteed end-to-end delays.

\section{References}

1. Le Boudec, J.Y., Thiram, P. : Network Calculus A Theory of Deterministic Queuing Systems for the Internet. Lecture Notes in Computer Science, Vol. 2050. Springer Verlag, (2001)

2. Cruz, R.L.: A Calculus for Network Delay, Part I : Network Elements in Isolation. IEEE Transactions on Information Theory, Vol. 37 no. 1. January(1991) 114-131

3. Ahlswede, R., Cai, N., Li, S.-Y.R., Yeung, R.W.: Network Information Flows. IEEE Transactions on Information Theory, Vol. 46. July(2000) 1204-1216

4. Y. Ma, W. Li, P. Fan, and X. Liu, "Queuing Model and Delay Analysis On Network Coding," in ISCIT, October 2005.

5. Eryilmaz, A., Ozdaglar, A., Médard, M.: On Delay Performance Gains from Network Coding. invited paper, Proceedings of the Conference on Information Sciences and Systems (CISS). (2006) 